\title{
Probing the structure of entanglement with entanglement moments
}

\author{
Justin H. Wilson ${ }^{\mathrm{a}}$, Joe Mitchell ${ }^{\mathrm{a}, *}$, Victor Galitski ${ }^{\mathrm{a}}$ \\ ${ }^{a}$ Joint Quantum Institute and Condensed Matter Theory Center, Department of Physics, University of Maryland, College Park, Maryland 20742-4111, USA
}

\begin{abstract}
We introduce and define a set of functions on pure bipartite states called entanglement moments. Usual entanglement measures tell you if two systems are entangled, while entanglement moments tell you both if and how two systems are entangled. They are defined with respect to a measurement basis in one system (e.g., a measuring device), and output numbers describing how a system (e.g., a qubit) is entangled with that measurement basis. The moments utilize different distance measures on the Hilbert space of the measured system, and can be generalized to any $N$-dimensional Hilbert space. As an application, they can distinguish between projective and non-projective measurements. As a particular example, we take the Rabi model's eigenstates and calculate the entanglement moments as well as the full distribution of entanglement.
\end{abstract}

Keywords: D. Entanglement; D. Projective Measurement; D. Harmonic Analysis

PACS: 03.65.Ud, 03.67.Mn

\section{Introduction}

Quantifying entanglement has been of interest since Bell showed that this uniquely quantum feature was available for experimental verification [1-4]. Since Bell, we have seen an explosion of potential applications in quantum information [5] and computation [6] as well as a whole body of theory to address the quantification of entanglement [7]. There are many measures of entanglement for pure states and mixed state [7] which take as input a state and outputs a number telling you, very roughly speaking, how entangled a state is. However, these measures just tell one if a state is entangled, but not how it is entangled: two very different states can give the same number. To address this, we define a new set of functions called entanglement moments. (While we call these entanglement "moments", they are not moments in the usual sense of distributions.) These quantities can tell us not only if and by how much a state is entangled but also how the distribution of entanglement looks by telling us how "clumpy" our distribution is.

For example, if we have a qubit entangled with another system and we make measurements on that other system, we will get a distribution of qubit states on the Bloch sphere. Two such examples are shown in Fig. 1. We would like a measure that can distinguish these two distributions - both of which have the same entanglement as given by the usual entanglement measures such as concurrence $[8,9]$.

As an application, the property of entanglement moments to describe how the system is entangled allows them to characterize measurements from weak to strong/projective measurements $[10,11]$. This uses the prescription for quantum measurement where the apparatus is treated quantum mechanically, becoming entangled with the system and mediating the collapse of

\footnotetext{
${ }^{*}$ Corresponding author; Phone +14053012659

Email address: jmitch3@umd.edu (Joe Mitchell)
}
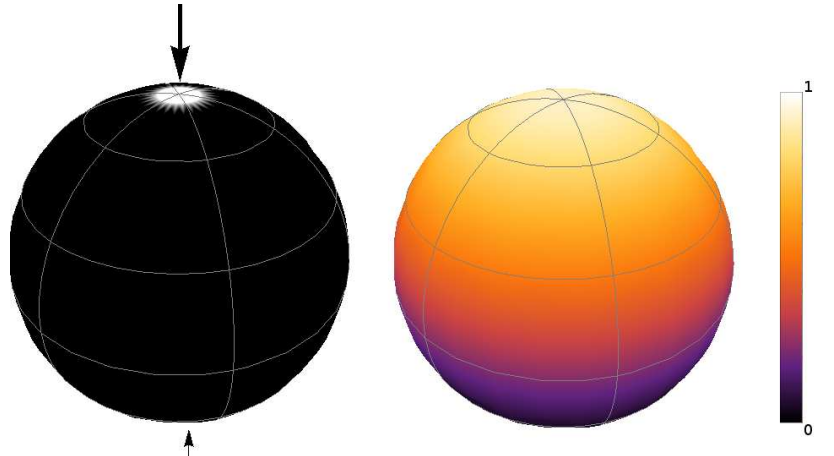

Figure 1: (Color online) Shown are two different distributions of entanglement on the Bloch sphere with the same concurrence. The left consists of two delta functions, on the north and south poles, with possibly different magnitudes (the arrow sizes are proportional to the strength of the delta functions). The right is a smoother distribution. Entanglement moments can distinguish them.

the wave function [12]. This phenomenon has been exploited to understand the measurement process in the lab in terms of finite strength quantum measurement (see for instance [13-15]). In this situation, a measuring device is entangled with another system, and making measurements on the device indirectly probes the second system in what may be a non-projective way. Considering the apparatus and system, a projective measurement corresponds to a very "clumpy" distribution in the system's Hilbert space while a non-projective measurement would be more evenly distributed. The entanglement moments can tell the difference between these two distributions and hence between projective and certain non-projective measurements.

To illustrate a physically relevant application of entanglement moments, we analyze the Rabi model [16]. This model shows up in many areas of physics including but not limited to circuit QED [17], cavity QED [18, 19], photonics [20], and 
flux qubits [21]. It comes into play when a qubit and a harmonic oscillator interact, and hence it finds its way into many of the approaches to quantum computation [22]. While entanglement in the Rabi model has been studied before [23], we show how the entanglement moments track the distribution of qubit states in particular eigenstates of the system - demonstrating how the moments discriminate between projective and non-projective measurements. The results are obtained numerically from the exact solution recently found by Braak [24]. In addition, the distribution of qubit states shows one way in which the Rabi model can give qualitatively different results from the Jaynes-Cummings model such as the non-monotonic behavior of entanglement with respect to the interaction strength (seen in Fig. 5).

In this article, we first define entanglement moments for two systems $A$ and $B$ where $A$ is the measured system (Sec. 2). Then, without specifying the system $B$ doing the measurement, we analyze the entanglement moments when our measured system $A$ is a qubit restricted to $S^{1}$ on the Bloch sphere (Sec. 2.1), when $A$ is the entire Bloch sphere (Sec. 2.2), and when $A$ is an arbitrary $N$-dimensional Hilbert space (Sec. 2.3). Throughout, we analyze the expressions with some informative examples. And finally, we apply the analysis to the eigenstates of the exactly solvable Rabi model (Sec. 3).

\section{Theoretical methods}

In order to address this question of "how" two systems can be entangled, we lift one of the requirements of entanglement measures: that the measure must be invariant under local unitary transformations [7]. To understand why we need to lift this requirement in order to get at the nature of the entanglement, consider a qubit $A$ coupled to any system $B$ with $|\Psi\rangle=$ $|\uparrow\rangle \otimes\left|\phi_{1}\right\rangle+|\downarrow\rangle \otimes\left|\phi_{2}\right\rangle$. Given any basis for $B,\{|1\rangle,|2\rangle, \ldots,|N\rangle\}$, we can always perform a unitary operation, $U$, on the Hilbert space of $B$ such that $U\left|\phi_{1}\right\rangle=a|1\rangle$ and $U\left|\phi_{2}\right\rangle=b|1\rangle+c|2\rangle$. This unitary operation has destroyed the information describing how the basis is entangled with our qubit. While it is true that only two states at any given time are entangled with a qubit, rarely can an experiment know what those two states are a priori. On the other hand, we must take into account the converse of this: We can rotate $a|1\rangle$ and $b|1\rangle+c|2\rangle$ into any two vectors (such that $\left\langle\phi_{1} \mid \phi_{2}\right\rangle=a^{*} b$ ). Thus, the choosing of a basis must have some physical relevance and hence we call it the measurement basis.

To construct these entanglement moments, consider systems $A$ and $B$ and let $\{|1\rangle,|2\rangle, \ldots,|N\rangle\}$ be the measurement basis in system $B$. We can write a general state vector as

$$
|\Psi\rangle=\sum_{i}\left|\psi_{i}\right\rangle \otimes|i\rangle,
$$

where $\left|\psi_{i}\right\rangle$ are unnormalized vectors in the Hilbert space of $A$. Defining the weight of the vector by $\left|\psi_{i}\right|^{2} \equiv\left\langle\psi_{i} \mid \psi_{i}\right\rangle$, we write the expression for $n$th entanglement moment as

$$
C_{(n)}^{2}=\mathcal{N}_{n} \sum_{i, j}\left|\psi_{i}\right|^{2} d_{(2 n)}^{2}(i, j)\left|\psi_{j}\right|^{2},
$$

where for the normalized $\left|\tilde{\psi}_{i}\right\rangle=\left|\psi_{i}\right\rangle /\left|\psi_{i}\right|$

$$
d_{(2 n)}^{2}(i, j)=1-\left|\left\langle\tilde{\psi}_{i} \mid \tilde{\psi}_{j}\right\rangle\right|^{2 n},
$$

and the quantity $\mathcal{N}_{n}$ normalizes the maximal value of $C_{(n)}^{2}$ to unity - we will specify its value for specific cases later. The quantity $d_{(2 n)}^{2}(i, j)$ is a distance function on the Hilbert space of system $A$. If $n=1$, we get the Hilbert-Schmidt distance measure (which leads to the Fubini-Study metric), and if we let $n \rightarrow \infty$, we get the trivial distance measure. The presence of the distance measure is to quantify how system $A$ changes as we measure system $B$; if system $A$ changes upon measurement of system $B$, we know they are entangled (for pure states, as considered here - this breaks down for mixed states). Therefore, a state $|\Psi\rangle$ is separable if and only if each entanglement moment is zero.

For the specific value $n=1$, we actually reproduce IConcurrence [9] in general (or just concurrence in the case of a qubit):

$$
C_{(1)}^{2}=\mathcal{N}_{1}\left[1-\operatorname{tr} \varrho_{A}^{2}\right],
$$

where $\varrho_{A}$ is the reduced density matrix of system $A$ and $\operatorname{tr} \varrho_{A}^{2}$ is the order-2 Rényi entropy [25]. Thus, the quantity $C_{(1)}^{2}$ is invariant under all local unitary transformation. Not only is this quantity I-Concurrence, but it begins with a clearer, geometric, and intuitive definition (Eq. (2)).

While we are mostly concerned with finite-dimensional Hilbert spaces in this paper, Eq. (2) can be applied to continuous variables and even Gaussian states.

\subsection{Entanglement momenuts on $S^{1}$}

To illustrate what happens when $n>1$, we first assume that not only is system $A$ on the Bloch sphere but that our states are constrained to be on the great circle $S^{1}$ defined by the $y$-axis. (This example is illustrative: we consider the entire Hilbert space in Secs. 2.2 and 2.3.) We can define the distribution of the entanglement $\rho: S^{1} \longrightarrow[0, \infty)$ such that $\rho(\theta)$ is the probability distribution of states in the Hilbert space of $A$ given measurements in $B$ with a particular basis (each $\theta$ corresponds to a particular state in Hilbert space). This allows us to rewrite the entanglement moments as

$$
C_{(n)}^{2}=\mathcal{N}_{n} \int_{-\pi}^{\pi} d \theta d \theta^{\prime} \rho(\theta) d_{(2 n)}^{2}\left(\theta, \theta^{\prime}\right) \rho\left(\theta^{\prime}\right) .
$$

(In the case of a countable number of $\left|\psi_{i}\right\rangle$ 's, $\rho(\theta)$ is a sum of delta functions.) In this representation, we know the exact form of the distance measure $d_{(2 n)}^{2}\left(\theta, \theta^{\prime}\right)=1-\cos ^{2 n}[(\theta-$ $\left.\left.\theta^{\prime}\right) / 2\right]$. With simple trigonometric identities and the normalization $\int_{-\pi}^{\pi} d \theta \rho(\theta)=1$, we obtain

$$
\begin{aligned}
C_{(n)}^{2}=\mathcal{N}_{n}\left[1-\frac{1}{2^{2 n}}\left(\begin{array}{c}
2 n \\
n
\end{array}\right)\right. & \\
& \left.-\frac{2}{2^{2 n}} \sum_{k=1}^{n}\left(\begin{array}{c}
2 n \\
n-k
\end{array}\right)\left|\int_{-\pi}^{\pi} d \theta \rho(\theta) e^{i k \theta}\right|^{2}\right],
\end{aligned}
$$


with normalization $\mathcal{N}_{n}\left[S^{1}\right]=\left(1-2^{-2 n}\left(\begin{array}{c}2 n \\ n\end{array}\right)\right)^{-1}$. Note that in general the distribution is nontrivial to calculate (e.g. going from measurement basis to Bloch sphere coordinates can involve a multivalued, nonlinear transformation), while entanglement moments are always described by Eq. (2), giving partial information about the distribution.

These entanglement moments are picking up the features of this distribution in terms of its Fourier components - the distance functions are diagonal in this basis. The $n$th moment decreases from unity for each Fourier coefficient - up to the $n$th that is non-zero. Corollary to this, since $C_{(1)}^{2}$ is invariant under local unitary transformations, the norm of the first Fourier component will remain the same no matter what measurement basis is chosen (this generalizes to $l=1$ for the spherical harmonics and the first harmonic in $\mathbb{C} P^{N-1}$, see Sec. 2.3).

Consider the equation for entanglement moments Eq. (2). As we increase $n$, the distance measures interpolate between the Cartesian distance on the circle to the trivial distance measure (defined such that ever point is a distance 1 away from every other point). If we now consider a state localized near the north and south poles, the points near the north pole are roughly a distance 1 from all points near the south pole for all distance measures $d_{(2 n)}^{2}$. Thus, each $C_{(n)}^{2}$ decreases solely because the normalization $\mathcal{N}_{n}$ decreases. In this way, a decreasing of the moments is indicative of there being more than one "clump" in the entanglement distribution. Hence, if measuring system $B$ corresponds to a projective measurement on $A$, one should see the entanglement moments all decrease.

\subsection{Generalization to the Bloch sphere}

If we know our system is highly entangled, we can use the moments to tell if measuring system $B$ will correspond to a projective measurement on system $A$. To illustrate this, we step from $S^{1}$ to the entire Bloch sphere $S^{2}$. Going through a similar procedure to the previous section, we obtain

$$
C_{(n)}^{2}=\frac{n+1}{n} \int_{S^{2}} d \mathbf{n} d \mathbf{n}^{\prime} \rho(\mathbf{n}) d_{(2 n)}^{2}\left(\mathbf{n}, \mathbf{n}^{\prime}\right) \rho\left(\mathbf{n}^{\prime}\right),
$$

and using the addition theorem for spherical harmonics:

$$
C_{(n)}^{2}=1-\frac{4 \pi}{n} \sum_{l=1}^{n} \frac{\left(\begin{array}{c}
2 n+1 \\
n-l
\end{array}\right)}{\left(\begin{array}{c}
2 n+1 \\
n
\end{array}\right)} \sum_{m=-l}^{l}\left|\int_{S^{2}} d \mathbf{n} \rho(\mathbf{n}) Y_{l m}^{*}(\mathbf{n})\right|^{2} .
$$

We see that instead of Fourier coefficients, we have harmonics: $\sum_{m}\left|\int_{S^{2}} \rho Y_{l m}^{*}\right|^{2}$.

\subsection{Generalization to $\mathbb{C} \mathrm{P}^{N-1}$}

The type of analysis done in the previous two sections is generalizable to any finite dimensional Hilbert space coupled to another Hilbert space. As we have assumed, we have a bipartite system where the Hilbert space is the direct product of two other Hilbert spaces $\mathcal{H}=\mathcal{H}_{A} \otimes \mathcal{H}_{B}$. We can write states $|\Psi\rangle \in \mathcal{H}$ in terms of an orthonormal basis of $\mathcal{H}_{B},\left\{|1\rangle,|2\rangle, \ldots,\left|N_{B}\right\rangle\right\}$ (in principle this basis need not be finite or even countable), and (unnormalized) vectors $\left|\psi_{i}\right\rangle \in \mathcal{H}_{A}$,

$$
|\Psi\rangle=\sum_{i}\left|\psi_{i}\right\rangle \otimes|i\rangle
$$

The vector $|\Psi\rangle$ is normalized $\langle\Psi \mid \Psi\rangle=1$ while $\left\langle\psi_{i} \mid \psi_{i}\right\rangle \leq 1$ in general.

Let system $\mathcal{H}_{A}$ be an arbitrary $N$-dimensional Hilbert space. The space of normalized vectors is $S^{2 N-1}$, but there is a U(1) gauge freedom in the distance measures given by Eq. (3), so the space is actually $\mathbb{C P}^{N-1}=S^{2 N-1} / \mathrm{U}(1)$. This is in fact a Hopf fibration [26] from $S^{2 N-1}$ to $\mathbb{C P}^{N-1}$ over the U(1) fiber. As with the other cases considered in the previous sections, we $\operatorname{map}\left\langle\psi_{i} \mid \psi_{i}\right\rangle$ onto the function $\rho: \mathbb{C P}^{N-1} \longrightarrow[0, \infty)$.

The entanglement moments are then given by

$$
C_{(n)}^{2}=\mathcal{N}_{n} \int_{\mathbb{C} \mathrm{P}^{N-1}} d \mu(z) \int_{\mathbb{C} \mathrm{P}^{N-1}} d \mu(w) \rho(z) d_{(2 n)}^{2}(z, w) \rho(w),
$$

and the distance functions are known:

$$
d_{(2 n)}^{2}(z, w)=1-\left|\sum_{i} z_{i}^{*} w_{i}\right|^{2 n} .
$$

The distance function can be considered as a function on the angle set, so that we write

$$
d_{(2 n)}^{2}(z, w)=d_{(2 n)}^{2}\left(2\left|\sum_{i} z_{i}^{*} w_{i}\right|^{2}-1\right) .
$$

Since Eq. (12) is an $n$th ordered polynomial in $2\left|\sum_{i} z_{i}^{*} w_{i}\right|^{2}-1$, we can expand it into Jacobi polynomials, $P_{k}^{(N-2,0)}\left(2\left|\sum_{i} z_{i}^{*} w_{i}\right|^{2}-\right.$ 1).

Now we need to use an addition formula for complex projective space as derived by [27-29]. To develop the formula, we should write the space of functions, $L^{2}\left(\mathbb{C P}^{N-1}\right)$, as a direct sum of orthogonal subspaces in the following way. Dividing into the spaces of spherical harmonics, we have $L^{2}\left(S^{2 N-1}\right)=$ $H_{1}(2 N) \oplus H_{2}(2 N) \oplus \cdots$, where $H_{m}(2 N)$ is the finite-dimensional vector space of harmonic polynomials homogeneous of degree $m$ of $2 N$ real variables that are restricted to $S^{2 N-1}$. These should be further restricted to those that are just $\mathrm{U}(1)$ invariant since $\mathrm{CP}^{N-1}=S^{2 N-1} / \mathrm{U}(1)$. With this restriction, we follow the notation of [30] and write

$$
L^{2}\left(\mathbb{C P}^{N-1}\right)=H_{(0,0)}(N) \oplus H_{(1,1)}(N) \oplus H_{(2,2)}(N) \oplus \cdots,
$$

where $H_{(m, m)}(N)$ are just the U(1) invariant parts of $H_{m}(2 N)$.

Given this, we now state the addition theorem as written in [29]. Let $d_{k, N}=\operatorname{dim} H_{(k, k)}(N)$ and $\left\{s_{k j}\right\}_{j=1}^{d_{k, N}}$ be an orthonormal basis in the space $H_{(k, k)}(N)$. Then the Jacobi polynomials become

$$
\begin{aligned}
P_{k}^{(N-2,0)}\left(2\left|\sum_{i} z_{i}^{*} w_{i}\right|^{2}-1\right) & \\
& =\frac{1}{d_{k, N}}\left(\begin{array}{c}
k+N-2 \\
k
\end{array}\right) \sum_{j=1}^{d_{k, N}} s_{k j}^{*}(z) s_{k j}(w) .
\end{aligned}
$$


Note that we can also calculate $d_{k, N}$ from formulae given in [29]. It is

$$
d_{k, N}=\frac{2 k+N-1}{N-1}\left(\begin{array}{c}
k+N-2 \\
k
\end{array}\right)^{2} .
$$

Just as before, we can expand our the distance function in terms of $P_{k}^{(N-2,0)}\left(2\left|\sum_{i} z_{i}^{*} w_{i}\right|^{2}-1\right)$, then expand that by the addition theorem and obtain

$$
C_{(n)}^{2}=\mathcal{N}_{n}\left[1-\sum_{k=0}^{n} \frac{\left(\begin{array}{c}
2 n+N-1 \\
n-k
\end{array}\right)}{\left(\begin{array}{c}
n+N-1 \\
n
\end{array}\right)\left(\begin{array}{c}
2 n+N-1 \\
n
\end{array}\right)}\|\rho\|_{H_{(k, k)}(N)}^{2}\right],
$$

where

$$
\|\rho\|_{H_{(k, k)(N)}}^{2}=\sum_{j=1}^{d_{k, N}}\left|\int_{\mathbb{C P}^{N-1}} d \mu(z) \rho(z) s_{k j}^{*}(z)\right|^{2}
$$

is the norm of the distribution $\rho$ in the finite subspace $H_{(k, k)}(N)$ - i.e., the norm in the $k$ th harmonic. So the $n$th entanglement moment captures the information about the 1 st through $n$th harmonic of the distribution.

Proper normalization of our distribution gives us $\|\rho\|_{H_{(0.0)}(N)}^{2}=$ 1 , since $H_{(0,0)}(N)$ is the space of constant functions. We can read off the normalization as

$$
\mathcal{N}_{n}\left[\mathbb{C P}^{N-1}\right]=\frac{\left(\begin{array}{c}
n+N-1 \\
n
\end{array}\right)}{\left(\begin{array}{c}
n+N-1 \\
n
\end{array}\right)-1} .
$$

This entire analysis reduces to the case of a Bloch sphere for $N=2$, and we reproduce the Bloch sphere formula Eq. (8) with the proper normalization

$$
\mathcal{N}_{n}\left[S^{2}\right]=\frac{n+1}{n} .
$$

\section{Results and Discussion}

In the following, we give examples of entanglement moments applied when our states are confined to the great circle $S^{1}$ and to the Bloch sphere $S^{2}$, in addition to a more substantial application to the Rabi model.

\subsection{Examples on $S^{1}$}

For further illustration, consider two distributions on $S^{1}$ :

$$
\begin{aligned}
& \rho_{1}(\theta)=\frac{1}{2}\left[\left(1+e^{-s}\right) \delta(\theta)+\left(1-e^{-s}\right) \delta(\theta-\pi)\right] \\
& \rho_{2}(\theta)=\frac{1}{2 \pi} \sum_{k=-\infty}^{\infty} e^{-k^{2} s} e^{i k \theta},
\end{aligned}
$$

where $s$ is an arbitrary parameter, $\rho_{1}(\theta ; s=0)=\rho_{2}(\theta ; s=$ $0)=\delta(\theta)$, and for every $s$, they give the same entanglement $C_{(1)}^{2}\left[\rho_{1}\right]=C_{(1)}^{2}\left[\rho_{2}\right]$. These are qualitatively like the two distributions in Fig. 1 except on $S^{1}$ instead of $S^{2}$. The first is a projective measurement, interpolating between the unentangled state at $s=0$ and equal probable projection as $s \rightarrow \infty$.

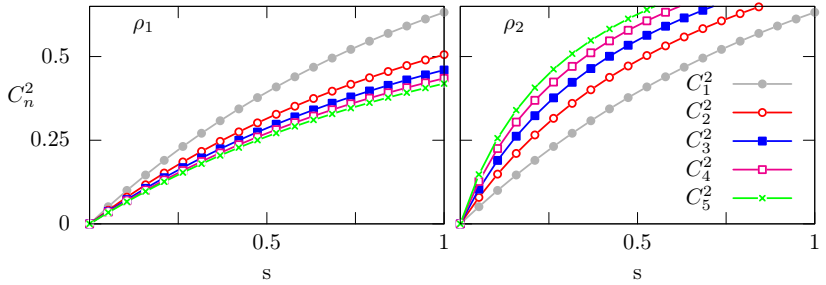

Figure 2: (Color online) Entanglement moments for distributions $\rho_{1}$ and $\rho_{2}$ from Eq. (20) on the circle $S^{1} . \rho_{1}$ changes from localized to the north pole to equally localized to north and south, while $\rho_{2}$ delocalizes from the north pole to cover the entire circle as $s \rightarrow \infty$. As $s \rightarrow \infty$, the moments of $\rho_{2}$ will limit to 1 , while those of $\rho_{1}$ will be less.

The second is a state localized around $\theta=0$, delocalizing as $s$ increases to eventually cover the whole circle. Plotting the higher entanglement moments in Fig. 2, we see a stark contrast. For $\rho_{1}$, all moments get smaller as we increase $n$, and for $\rho_{2}$, they get larger. Consider the mode decomposition Eq. (6): the higher modes in $\rho_{2}(\theta)$ are exponentially suppressed; while in $\rho_{1}(\theta)$, half of the higher modes stay constant.

The entanglement moments generated by the two functions in Eq. (20) depend differently on $n$. For $\rho_{1}$, we can simply perform the integration and we easily obtain

$$
C_{(n)}^{2}\left[\rho_{1}\right]=\frac{1}{2}\left(1-e^{-2 s}\right)\left(1+\frac{1}{\sqrt{\pi n}}\right) .
$$

For $\rho_{2}$, we can use the approximation $\left(\begin{array}{c}2 n \\ n-k\end{array}\right) \sim\left(\begin{array}{c}2 n \\ n\end{array}\right) e^{-k^{2} / n}$ and expand Eq. (6) to obtain for large $n$,

$$
C_{(n)}^{2}\left[\rho_{2}\right]= \begin{cases}1-\left(\frac{1}{\sqrt{s n+1}}-\frac{1}{\sqrt{\pi n}}\right), & s \ll 1, \\ 1-\frac{2}{\sqrt{\pi n}} e^{-2 s}, & s \gg 1 .\end{cases}
$$

$C_{(n)}^{2}\left[\rho_{1}\right]$ decreases as $1 / \sqrt{n}$, while $C_{(n)}^{2}\left[\rho_{2}\right]$ increases as $1 / \sqrt{n}$. A similar analysis holds for $S^{2}$ and $\mathbb{C P}^{n-1}$.

\subsection{Bloch sphere}

Fig. 3 shows the entanglement moments for three distributions on $S^{2}$ that all have the same, maximal concurrence (i.e. $C_{(1)}^{2}=1$ ): (1) evenly distributed about the sphere, (2) evenly localized along the equator, and (3) localized to the north and south poles. Case 1 remains at 1 , case 2 dips then returns to 1 , and case 3 asymptotes to $\frac{1}{2}$; each indicates their level of localization. Considering case 2 , it rises back to 1 because no state in its distribution is localized to a set of measure zero - if we go to our original expression for entanglement and let $n \rightarrow \infty$, then $d_{(\infty)}^{2}(i, j)=1$ if $\left|\tilde{\psi}_{i}\right\rangle$ and $\left|\tilde{\psi}_{j}\right\rangle$ are not the same and it is 0 if they are.

\subsection{Rabi model}

For a more in depth example, we consider a qubit $\left(\mathcal{H}_{A}\right)$ and a harmonic oscillator $\left(\mathcal{H}_{B}\right)$ described by the Rabi Hamiltonian

$$
H_{\text {Rabi }}=\omega a^{\dagger} a+g \sigma_{x}\left(a+a^{\dagger}\right)+\frac{1}{2} \Delta \sigma_{z},
$$

where $a\left(a^{\dagger}\right)$ is the annihilation (creation) operater, $\sigma_{x}$ and $\sigma_{z}$ are the $x$ and $z$ Pauli matrices respectively, and $\omega, g$, and $\Delta$ are 


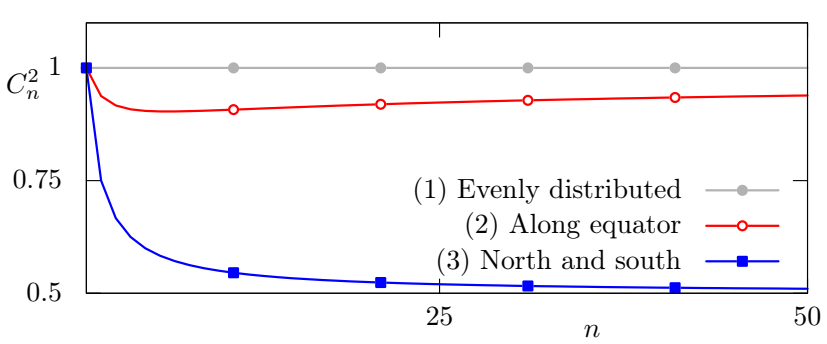

Figure 3: (Color online) Entanglement moments for distributions on the Bloch sphere. All have maximal concurrence $C_{(1)}^{2}=1$, but the moments of the more localized distributions deviate greatly. The $n \rightarrow \infty$ limit of the distribution localized to north and south poles reveals its pointlike nature.

constants (frequency of the oscillator, coupling, and Zeeman splitting, respectively).

Braak [24] solved equation (23) for the eigenstates. The eigenvalues can be calculated from $E_{n}^{ \pm}=\xi_{n}^{ \pm}-g^{2} / \omega$ where $\xi_{n}^{ \pm}$ solve the secular equation

$$
0=G_{ \pm}\left(\xi_{n}^{ \pm}\right)=\sum_{m=0}^{\infty} K_{m}\left(\xi_{m}^{ \pm}\right)\left[1 \mp \frac{\Delta}{\xi_{m}^{ \pm}-m \omega}\right]\left(\frac{g}{\omega}\right)^{2}
$$

and the coefficients $K_{m}(\xi)$ satisfy

$$
\begin{gathered}
m K_{m}=f_{n-1}(\xi) K_{m-1}-K_{m-2}, \\
K_{0}=1, \quad K_{1}(\xi)=f_{0}(\xi), \\
f_{m}(\xi)=\frac{2 g}{\omega}+\frac{1}{2 g}\left(m \omega-\xi+\frac{\Delta^{2}}{\xi-m \omega}\right) .
\end{gathered}
$$

The unnormalized eigenstates, written in Bargmann space [31], are

$$
\begin{aligned}
\phi_{n}^{ \pm}(z)=e^{g z} \sum_{n=0}^{\infty} K_{n}\left(\xi_{n}^{ \pm}\right)(-z & +g)^{n} \\
& = \pm e^{g z} \sum_{n=0}^{\infty} K_{n}\left(\xi_{n}^{ \pm}\right) \Delta \frac{(z+g)^{n}}{\xi_{n}^{ \pm}-n} .
\end{aligned}
$$

Taking normalization into account, we can obtain

$$
C_{(1)}^{2}\left[\left|\Psi_{n}^{ \pm}\right\rangle\right]=1-\left(\frac{\sum_{n=0}^{\infty} n ! K_{n}\left(\xi_{n}^{ \pm}\right)^{2} \frac{\Delta}{\xi_{n}^{ \pm}-n}}{\sum_{n=0}^{\infty} n ! K_{n}\left(\xi_{n}^{ \pm}\right)^{2}}\right)^{2} .
$$

With our measurement basis as the eigenbasis for the operator $\hat{x}=\frac{1}{\sqrt{2}}\left(a+a^{\dagger}\right)$, we can write a vector in this Hilbert space as

$$
|\psi\rangle=\int d x|\psi(x)\rangle \otimes|x\rangle,
$$

where $|\psi(x)\rangle$ is a vector on the Bloch sphere. This set of vectors can be mapped onto a distribution $\rho$ on the Bloch sphere.

Now consider explicitly the eigenstates of Eq. (23). They can be labeled by an integer and \pm as shown in the exact solution given by Braak [24]. These states, $|n, \pm\rangle$, only live on a circle $S^{1}$ of the Bloch sphere due to the exclusion of $\sigma_{y}$ from the

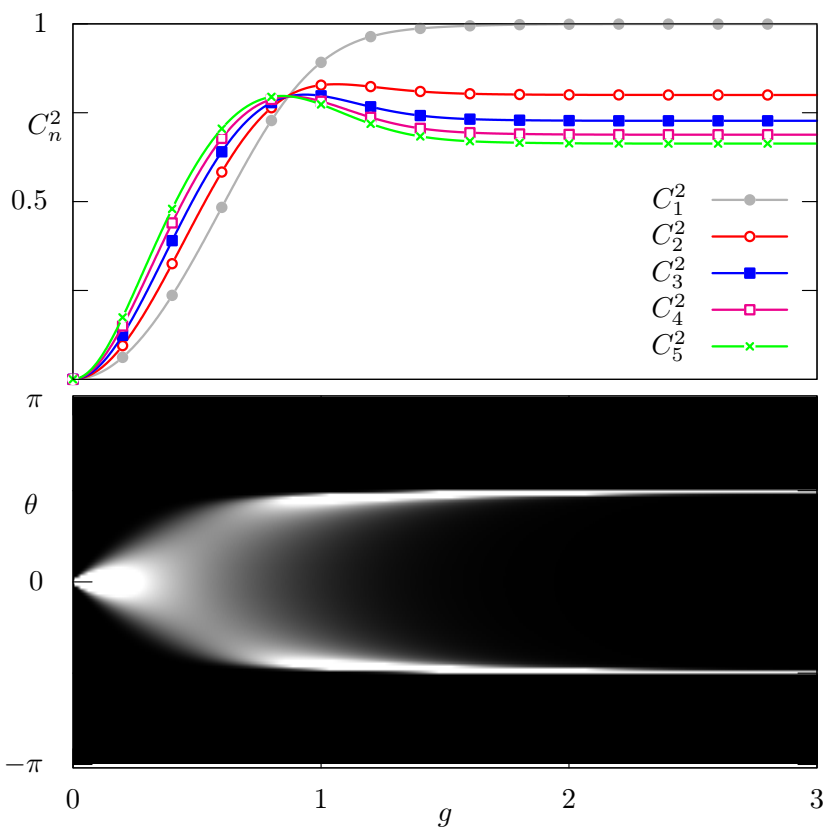

Figure 4: (Color online) Rabi model ground state with $\Delta=0.3$. Top: Entanglement moments. Note that the crossover corresponds roughly with the distribution forming into two nonoverlapping localized points. Bottom: Plot of the distribution along the relevant circle on the Bloch sphere.
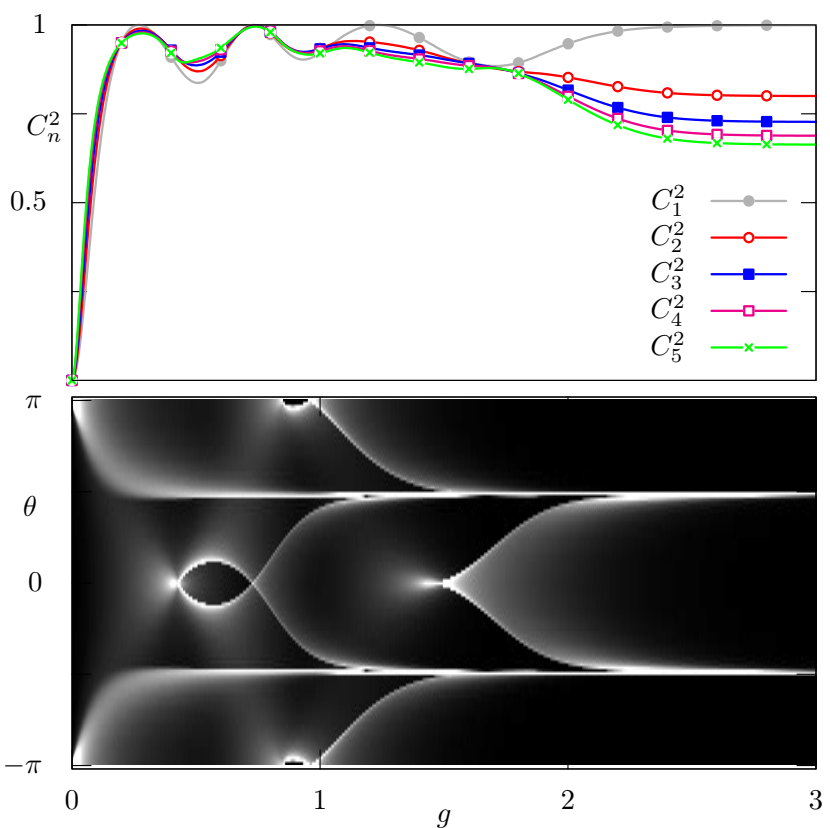

Figure 5: (Color online) The sixth excited state of the Rabi model with $\Delta=$ 0.3 . The concurrence, $C_{(1)}^{2}$, does not hold the information necessary to deal with such a complicated state. Top: Entanglement moments. Bottom: Plot of the distribution along the relevant circle on the Bloch sphere. Notice how the entanglement is non-monotic with respect to the coupling $g$. 
Hamiltonian. As such, we use Eq. (5) for the entanglement moments. The entanglement moments and full distributions for $\Delta=0.3$ are plotted for the ground state in Fig. 4 and for the sixth excited state in Fig. 5.

Notice in these figures how the distribution changes with $g$ for a given eigenstate. For large $g$, the higher moments asymptote to a value less than 1 while $C_{(1)}^{2} \rightarrow 1$. This represents the localization described in Fig. 3 as well as how a measurement in $\hat{x}$ corresponds to a projective measurement in $\sigma_{x}$ of the qubit. On the other hand, the figures show other instances where $C_{(1)}^{2}=1$ along with other entanglement moments, and in those cases the states are more evenly distributed about the circle. In fact when we see moments rise for higher values of $n$, we know the state is becoming more evenly distributed just as in the case of Fig. 2. The cross-over from the moments getting larger as $n$ increases to the point where they start decreasing with $n$ represents the cross-over from non-projective to projective-like measurements.

\section{Conclusion}

While the full distribution $(\rho(\theta)$ in Eq. (5)) has all relevant information, the entanglement moments provide not only a calculational tool to probe the state without finding $\rho(\theta)$ in its entirety but also a numerical answer to the question: How projective is a measurement? At this point, the idea is simple: The decreasing of entanglement moments indicates projectiveness, but if there is still some finite spreading of the distribution, the moments will start to increase again (for example, see Figs. 2 and 3). The value $n$ at which this occurs characterizes how projective a measurement is.

Entanglement moments could also be used in various dynamical questions and in many other systems (such as the system considered in [32]). However, this paper has dealt with pure states of bipartite systems, and expanding this concept to mixed states would be valuable.

We have defined the new concept of entanglement moments. These moments contain and surpass traditional entanglement measures, describing not only if a system is entangled, but also how. Taken all together, they can qualitatively and quantitatively describe how projective a measurement is. As a practical example, we calculated these moments for eigenstates of the Rabi model, showing complex behavior for higher excited states.

This research was supported by DOE-BES-DESC0001911 (V.G. and J.M.) and the JQI-PFC (J.W.).

\section{References}

[1] J. S. Bell, On the einstein-podolsky-rosen paradox, Physics 1 (3) (1964) 195-200.

[2] J. S. Bell, On the problem of hidden variables in quantum mechanics, Rev. Mod. Phys. 38 (3) (1966) 447-452.

[3] A. Aspect, P. Grangier, G. Roger, Experimental tests of realistic local theories via bell's theorem, Phys. Rev. Lett. 47 (7) (1981) 460-463.

[4] H. M. Wiseman, S. J. Jones, A. C. Doherty, Steering, entanglement, nonlocality, and the einstein-podolsky-rosen paradox, Phys. Rev. Lett. 98 (14) (2007) 140402.
[5] A. K. Ekert, Quantum cryptography based on bells theorem, Phys. Rev. Lett. 67 (6) (1991) 661-663.

[6] R. Horodecki, M. Horodecki, K. Horodecki, Quantum entanglement, Rev. Mod. Phys. 81 (2) (2009) 865-942.

[7] M. B. Plenio, S. Virmani, An introduction to entanglement measures, Quant. Info. and Comput. 7 (1-2) (2007) 1-51.

[8] W. K. Wootters, Entanglement of formation of an arbitrary state of two qubits, Phys. Rev. Lett. 80 (10) (1998) 2245-2248.

[9] P. Rungta, V. Bužek, C. M. Caves, M. Hillery, G. J. Milburn, Universal state inversion and concurrence in arbitrary dimensions, Phys. Rev. A 64 (4) (2001) 042315.

[10] W. H. Zurek, Pointer basis of quantum apparatus: Into what mixture does the wave packet collapse?, Phys. Rev. D 24 (6) (1981) 1516-1525.

[11] W. H. Zurek, Environment-induced superselection rules, Phys. Rev. D 26 (8) (1982) 1862-1880.

[12] J. Von Neumann, Mathematical Foundations of Quantum Mechanics, Vol. 72 of Princeton landmarks in mathematics and physics, Princeton University Press, 1955.

[13] M. Brune, E. Hagley, J. Dreyer, X. Maître, A. Maali, C. Wunderlich, J. M. Raimond, S. Haroche, Observing the progressive decoherence of the "meter" in a quantum measurement., Physical Review Letters 77 (24) (1996) 4887-4890.

[14] R. Vijay, C. Macklin, D. H. Slichter, S. J. Weber, K. W. Murch, R. Naik, a. N. Korotkov, I. Siddiqi, Stabilizing rabi oscillations in a superconducting qubit using quantum feedback., Nature 490 (7418) (2012) 77-80.

[15] M. Hatridge, S. Shankar, M. Mirrahimi, F. Schackert, K. Geerlings, T. Brecht, K. M. Sliwa, B. Abdo, L. Frunzio, S. M. Girvin, R. J. Schoelkopf, M. H. Devoret, Quantum back-action of an individual variable-strength measurement., Science 339 (6116) (2013) 178-81.

[16] I. Rabi, On the process of space quantization, Phys. Rev. 49 (4) (1936) 324-328.

[17] T. Niemczyk, F. Deppe, H. Huebl, E. P. Menzel, F. Hocke, M. J. Schwarz, J. J. Garcia-Ripoll, D. Zueco, T. Hümmer, E. Solano, A. Marx, R. Gross, Circuit quantum electrodynamics in the ultrastrong-coupling regime, $\mathrm{Na}$ ture Physics 6 (10) (2010) 772-776

[18] M. Schiró, M. Bordyuh, B. Öztop, H. E. Türeci, Phase transition of light in cavity qed lattices, Phys. Rev. Lett. 109 (5) (2012) 053601

[19] D. Englund, A. Faraon, I. Fushman, N. Stoltz, P. Petroff, J. Vucković, Controlling cavity reflectivity with a single quantum dot., Nature 450 (7171) (2007) 857-861.

[20] A. Crespi, S. Longhi, R. Osellame, Photonic realization of the quantum rabi model, Phys. Rev. Lett. 108 (16) (2012) 163601.

[21] P. Forn-Díaz, J. Lisenfeld, D. Marcos, J. J. García-Ripoll, E. Solano, C. J. P. M. Harmans, J. E. Mooij, Observation of the bloch-siegert shift in a qubit-oscillator system in the ultrastrong coupling regime, Phys. Rev. Lett. 105 (23) (2010) 237001.

[22] T. Pellizzari, S. A. Gardiner, J. I. Cirac, P. Zoller, Decoherence, continuous observation, and quantum computing: A cavity qed model, Phys. Rev. Lett. 75 (21) (1995) 3788-3791.

[23] M.-f. Fang, P. Zhou, Quantum entropy and entanglement in the jaynescummings model without the rotating-wave approximation, Physica A 234 (1-2) (1996) 571-580.

[24] D. Braak, Integrability of the rabi model, Phys. Rev. Lett. 107 (10) (2011) 100401.

[25] I. Bengtsson, K. Zyczkowski, Geometry of Quantum States: An Introduction to Quantum Entanglement, Cambridge University Press, 2006.

[26] H. Hopf, ber die abbildungen der dreidimensionalen sphre auf die kugelffche, Mathematische Annalen 104 (1) (1931) 637-665.

[27] T. H. Koornwinder, The addition formula for jacobi polynomials, ii. the laplace type integral representation and the product formula, Tech. Rep. TW 133, Math. Centrum Amsterdam, Afd. Toegepaste Wiskunde (1972).

[28] T. H. Koornwinder, The addition formula for jacobi polynomials, iii. completion of the proof, Tech. Rep. TW 135, Math. Centrum Amsterdam, Afd. Toegepaste Wiskunde (1972).

[29] O. Shatalov, Isometric embeddings $\ell_{2}^{m} \rightarrow \ell_{p}^{n}$ and cubature formulas over classical fields, Ph.D. thesis, Technion - Israel Institute of Technology (2001).

[30] E. L. Grinberg, Spherical harmonics and integral geometry on projective spaces, Trans. Am. Math. Soc. 279 (1) (1983) 187.

[31] V. Bargmann, On a hilbert space of analytic functions and an associated integral transform part i, Commun. Pure Appl. Math. 14 (3) (1961) 187- 
214.

[32] J. H. Wilson, B. M. Fregoso, V. M. Galitski, Entanglement dynamics in a non-markovian environment: an exactly solvable model, Phys. Rev. B 85 (17) (2012) 174304. 